examined the public health burden of anogenital HPVCs and HSVCs among PLWH from a southeastern US HIV clinic.

Methods Electronic health records from the HIV Clinic at University of Alabama, Birmingham (01/2006-03/2018) were reviewed. Patients $\geq 18$ years at HIV diagnoses with $\geq 2$ clinical visits were analyzed. Incidence rates of HPVCs (cervical and vaginal/vulvar low and high grade squamous intraepithelial lesion (LSIL, HSIL) and cancers in women, penile cancers in men, warts, anal LSIL, HSIL, and cancers in both) and herpetic ulcers were calculated. Each condition was counted only once at its first diagnosis in the period. We used Joinpoint regression to estimate average annual percentage changes (AAPCs).

Results There were 1038 HPVCs, 546 HSVCs, and 3191 both condition-free, with mean ages: $38.3,39.6$, and 41.3 years, and median nadir CD4 counts: 243, 283, 323 cells $/ \mu \mathrm{L}$, respectively. Incidence of warts, anal LSIL, HSIL, and cancer were different between men $(189,252,44,26$ per 10,000 PYs) and women $(68,15,6,0$ per 10,000 PYs) $(p<0.0001$ for each). Racial disparities were observed in anal LSIL and cancer, cervical HSIL and cancer among whites (rates: 284, 28, 162, 50 per 10,000 PYs) and blacks (rates: 142, 14, 94, 15 per 10,000 PYs), respectively $(p<0.05$ for each). Incident ulcers were higher among women than men (260 vs 163 per 10,000 PYs) and blacks than whites (192 vs 183 per 10,000 PYs). Warts, anal HSIL and cancer, cervical LSIL and cancer increased significantly over time (AAPCs: 19.1, 25.3, 24.9, 13.0, 15.1\%, $\mathrm{P}<0.0001$ for each, respectively).

Conclusion HPVCs and HSVCs are common in the southeastern US PLWH, with substantial increases of warts, anal and cervical lesions and cancers. Better screenings are warranted in the high-risk population.

Disclosure No significant relationships.

\section{P846 BIOMARKERS OF HIV EXPOSURE AND CONDOMLESS RECEPTIVE ANAL SEX IN MEN WHO HAVE SEX WITH MEN USING SELF-COLLECTED RECTAL SWABS}

${ }^{1}$ Maria Lemos, ${ }^{2}$ Vijay Nandi, ${ }^{3}$ Joan Dragavon, ${ }^{1}$ Ira Fleming, ${ }^{1}$ Keertana Krishnan, ${ }^{2}$ Martin Musuruana, ${ }^{1}$ Madeline Kramer, ${ }^{1}$ Hayley Glantz, ${ }^{1}$ Michele Andrasik, ${ }^{4}$ Beryl Koblin, ${ }^{3}$ Robert Coombs, ${ }^{1} \mathrm{M}$ Mcelrath, ${ }^{2}$ Hong Tieu. ${ }^{1}$ Fred Huchinson Cancer Research Center, Vaccine and Infectious Disease Division, Seattle, USA; ${ }^{2}$ Lindsley F. Kimball Research Institute, New York Blood Center, Laboratory of Data Analytics, New York, USA; ${ }^{3}$ University of Washington, Departments of Laboratory Medicine, Seattle, USA; ${ }^{4}$ Lindsley F. Kimball Research Institute, New York Blood Center, Laboratory of Infectious Disease Prevention, New York, USA

10.1136/sextrans-2019-sti.890

Background Biomarkers of HIV exposure could help identify subpopulations at highest risk of HIV acquisition, to focus public health interventions and prevention strategies. This study assessed Y-chromosome single tandem repeat (YSTR) mixtures as biomarkers of receptive anal intercourse without condoms (RAIWC) among men who have sex with men (MSM). We also evaluated the feasibility of self-administered rectal swabs for detection of HIV virions to assess exposures.

Methods Thirty 18-to-50-year-old sexually active, HIV-seronegative MSM were enrolled in New York City. Participants answered daily sexual behavior questions via a mobile phone for 60 days, and were randomized to collecting self-administered rectal swabs daily or after every receptive anal intercourse (RAI) event. Blood collections, rapid HIV diagnostics, and counselling were performed at beginning and end of the study. YSTR mixtures were assessed in DNA from blood and 233 swabs from 20 participants reporting at least one RAIWC event. HIV exposure was measured by virion PCR in 171 swabs linked to reports of RAIWC.

Results As markers of partner's DNA, YSTR mixtures were found in $41 / 138(29.7 \%)$ of self-collected swabs linked to mobile reports of RAI. 15/83 (18\%) swabs collected after reporting abstinence, insertive sex or RAIWC had YSTR mixtures. No participant became HIV infected during the study, yet exposure to HIV was detected in six rectal swabs corresponding to two different participants. For one participant on pre-exposure prophylaxis (PrEP), rectal HIV virions were found after RAIWC with two different HIV positive partners not on antiretroviral therapy. For the second participant, rectal HIV exposures were identified after RAIWC with two different partners disclosing HIV negative status.

Conclusion YSTR mixtures in self-collected rectal swabs demonstrated $82 \%$ specificity but only $30 \%$ sensitivity to assess RAIWC. Detection of HIV exposure in self-collected swabs from two uninfected participant indicates it was possible to measure rectal HIV exposures in MSM.

Disclosure No significant relationships.

\section{P848 REVISITING ENFUVIRTIDE'S MECHANISM AND DESIGNING ITS ANALOG WITH IMPROVED ACTIVITY BY TARGETING TRIPLE SITES IN GP41}

${ }^{1}$ Shibo Jiang ${ }^{*},{ }^{1}$ Wei Xu, ${ }^{2}$ Jing Pu, ${ }^{2}$ Shan Su, ${ }^{2}$ Chen Hua, ${ }^{2}$ Xiaojie Su, ${ }^{2}$ Qian Wang, ${ }^{2}$ Lu Lu. ${ }^{1}$ Fudan University, School of Basic Medical Sciences, Shanghai, China; ${ }^{2}$ Shanghai Medical College of Fudan University, Shanghai, China

10.1136/sextrans-2019-sti.891

Background In the early of 1990's, several peptides overlapping gp41 CHR region, including SJ-2176, T20, and C34 were reported to have potent HIV-1 fusion inhibitory activity. T20 (generic name: enfuvirtide) was approved by the U.S. FDA as the first HIV-1 fusion inhibitory peptide-based antiHIV drug. However, its clinical application is limited because of its low potency and low genetic barrier to resistance. Furthermore, its mechanism of action is still elusive. Therefore, it is essential to define T20's mechanism of action, based on which a new analogous peptide with improved antiviral activity can be designed.

Methods The inhibitory activity of peptides on $6 \mathrm{HB}$ formation was tested in a temperature-controlled cell-cell fusion assay by flow cytometry using 6HB-specific mAb 2G8; on HIV-1 infection and fusion was assessed by p24 and cell-cell fusion assays. Interaction between different peptides or peptide and antibody was evaluated by ELISA.

Results T20 could inhibit 6HB formation at early, but not late, stage of HIV-1 fusion, while T1144 was effective at both stages. T20-SF is much more effective than T20 in binding to FP-P and inhibiting infection of HIV-1, including T20-resistant strains, and FP-P-mediated hemolysis.

Conclusion In the early of 1990's, several peptides overlapping gp41 CHR region, including SJ-2176 (residues 630-659), T20 (residues 638-673), and C34 (residues 628-661) were reported to have potent HIV-1 fusion inhibitory activity. T20 (generic name: enfuvirtide) was approved by the U.S. FDA as 
the first HIV-1 fusion inhibitory peptide-based anti-HIV drug. However, its clinical application is limited because of its low potency and low genetic barrier to resistance. Furthermore, its mechanism of action is still elusive. Therefore, it is essential to define T20's mechanism of action, based on which a new analogous peptide with improved antiviral activity against divergent HIV-1 strains, including those resistant to T20.

Disclosure No significant relationships.

\section{P850 GENDER IDENTIFY AND SELF-REPORTED HPV VACCINATION AMONG YOUTH LIVING WITH OR AT HIGH-RISK FOR HIV}

'Elizabeth Arnold*, ${ }^{2}$ Dallas Swendeman, ${ }^{2}$ Cameron Goldbeck, 'Kate Bridges, ${ }^{3}$ Sue Ellen Abdalian, ${ }^{4}$ Jeffrey Klausner. ${ }^{1}$ UT Southwestern Medical Center, Dallas, USA; ${ }^{2}$ University of California Los Angeles, Los Angeles, USA; ${ }^{3}$ Tulane University, New Orleans, USA; ${ }^{4}$ UCLA David Geffen School of Medicine and Fielding School of Public Health, Epidemiology, Los Angeles, USA

\subsection{6/sextrans-2019-sti.892}

Background Human Papillomavirus (HPV) is common and is linked to cancer in both males and females. Efforts are underway to increase uptake of the vaccine (Gardasil) that is now recommended for youth and adults up to age 45 . However, little is known about the role of gender identify on vaccination, particularly among youth at increased risk for contracting and spreading the virus.

Methods We analyzed data $(\mathrm{N}=1070)$ from a longitudinal study of high-risk HIV negative youth and youth living with HIV aged 12-24. Data were collected in Los Angeles, CA and New Orleans, LA from clinic and community settings. We examined self-reports of HPV vaccination as well as predictors of vaccination.

Results Cis-gender females (CGFs) had the highest proportion of youth reporting vaccination $(52.7 \%)$ followed by transgender youth (45.9\%), gender non-conforming youth (44.2\%), and MSM (39.0\%). Cis-gendered, heterosexual males (CGHMs) reported the lowest proportion (32.9\%). However, $18.8 \%$ of youth did not know if they had received the vaccine. In our multivariate logistic model examining predictors of vaccination, gender identity was only significant for CGHMs. Other significant variables included age, some education higher than a high school diploma or completing higher education compared to less than high school, ever being tested for Hepatitis C, currently having a healthcare provider, always using condoms, and Latino or other race/ethnicity compared to African-American.

Conclusion Results suggest that despite national efforts to increase the uptake of the vaccine, many youth at high-risk for HPV have not received it or did not know if they had. CGFs appear to have the highest uptake, but CGMs are a group for whom increased efforts targeting vaccination are needed. Demographic and health care factors may also impact vaccination rates. Research is needed on barriers to vaccination and new interventions that go beyond education and promotion such as policy initiatives.

Disclosure No significant relationships.
P851 PERFORMANCE DISK DIFFUSION METHOD IN IDENTIFYING GONOCOCCAL RESISTANCE

${ }^{1}$ Wenqi Xu*, ${ }^{1}$ Jingwei Liu, ${ }^{1}$ Xiaoyu Zhu, ${ }^{2}$ Yueping Yin. ${ }^{1}$ National Center for STD Control, Chinese Center for Disease Control and Prevention, Chinese Academy of Medical Sciences and Peking Union Medical College, Nanjing, China; ${ }^{2}$ National Center for STD Control, Chinese Center for Disease Control and Prevention, Nanjing, China; Institute of Dermatology, Chinese Academy of Medical Sciences and Peking Union Medical College, Nanjing, China

\subsection{6/sextrans-2019-sti.893}

Background Because of their simple and cost-effective characteristics, many products of disk diffusion tests are commanly used by microbiological laboratories and hospitals world-wide to determine the gonococcal susceptibility and guide clinical treatments. To evaluate the reliability of routine detection of antimicrobial susceptibility of Neisseria gonorrhoeae by disk diffusion method, six kinds of discs targeted at the specific antibiotics (penicillin, ceftriaxone, cefixime, tetracycline, ciprofloxacin and spectinomycin) from two domestic and one foreign manufacturer were included into the evaluation using agar dilution method as a reference method to validate the concordance between the two methods.

Methods According to CLSI 2018 (M100, 28 ${ }^{\text {th }}$ ed) standard, the antimicrobial sensitivity results of more than 100 clinical isolates and 6 reference strains were interpreted. The coincidence rates of the two methods were analyzed by calculating four indicators: categorical agreement (CA), very major errors (VME), major errors (ME), minus errors (MIE). In addition, the correlation between agar dilution and disk diffusion methods were analyzed by using breakpoints established by linear least-squares regression.

Results The results showed that the MIC values of the 6 antibiotics on reference strains determined by the two methods were within the reference ranges. No very major or major discrepancies were detected in the 6 antibiotics. The performance of disk diffusion method to detect gonococcal susceptibility indicted that 1) the coincidence rates in spectinomycin and ciprofloxacin were high $(\mathrm{CA} \geq 98 \%) ; 2$ ) disk dilution method could miss some penicillin-resistant strains; (3) the coincidence rate of ceftriaxone and cefixime was low $(\mathrm{CA}<80 \%)$, in which agar dilution method is needed to confirm the detection of non-sensitive strains.

Conclusion The domestic products for ciprofloxacin, tetracycline, spectinomycin, cefixime and ceftriaxone were as satisfactory as foreign ones but the domestic for penicillin was relatively poor in quality.

Disclosure No significant relationships.

\section{P852 EVALUATION OF PLEXPCR ${ }^{\varpi}$ VHS FOR DIAGNOSIS OF GENITAL LESIONS}

${ }^{1}$ Litty Tan*, ${ }^{1}$ Peter Njuguna, ${ }^{1}$ Simon Erskine, ${ }^{2}$ Janet Towns, ${ }^{2}$ Marcus Chen, ${ }^{3} J u l i a n$ Druce, ${ }^{3}$ Franca Azzato, ${ }^{3}$ Thomas Tran, ${ }^{1}$ Elisa Mokany. 'SpeeDx Pty Ltd., Sydney, Australia; ${ }^{2}$ Melbourne Sexual Health Centre, Melbourne, Australia; ${ }^{3}$ Victorian Infectious Diseases Reference Laboratory, Melbourne, Australia

10.1136/sextrans-2019-sti.894

Background Genital lesions can be caused by herpes simplex virus types 1 and 2 (HSV-1 and HSV-2), varicella zoster virus (VZV) and Treponema pallidum (TP). Clinical presentations 